can lead to a collective and individual accident prevention policy, with adequate personal protective equipment and work environment.

\section{P-473 THE INFLUENCE OF SEX ON THE ASSOCIATION BETWEEN CHIKUNGUNYA INFECTION AND DEPRESSIVE SYMPTOMS IN HEALTHCARE WORKERS}

${ }^{1}$ Fernando Feijó, Margarete Costa Helioterio, Fernanda De Oliveira Souza, Paloma de Sousa Pinho, Tânia Maria De Araújo, Guilherme Werneck. ${ }^{1}$ Federal University of Bahia, Brazil

\subsection{6/OEM-2021-EPI.343}

Introduction Chikungunya viruses are endemic in tropical countries like Brazil. Healthcare workers (HCW) are at risk of infection in endemic areas. Besides causing chronic joint pain, the infection is hypothesised to be associated with mental health problems such as depression. However, no studies investigated this association and its relationship with sex are available.

Objectives To evaluate the association between previous infection by Chikungunya and depressive symptoms in HCW, investigating the role of sex in this relationship.

Methods This is a cross-sectional study with a representative sample $(\mathrm{n}=343)$ of primary care and medium complexity HCW in the municipality of Santo Antônio de Jesus, Bahia, Brazil, in 2019. The DPP ZDC IgM/IgG rapid test was used to investigate recent infection (measured by $\operatorname{IgM}$ ) or previous infection (by $\operatorname{IgG}$ ) by the Chikungunya virus. The Patient Health Questionnaire (PHQ-9) was used to measure depressive symptoms. Poisson regression with robust variance estimation was used to estimate prevalence ratios (PR) and adjust for confounders. Stratified analyses by sex were performed.

Results The prevalence of recent or old Chikungunya infection was $8.9 \%$, while the prevalence of depressive symptoms was $22.4 \%$. Chikungunya infection was associated with a 2.05 (95\%CI: 1.31-3.22) times higher prevalence of depressive symptoms in the bivariate analysis. After adjusting for sex, age, education, skin color and sequelae of chronic pain, the association remained strongly positive $(\mathrm{PR}=1.74$ (95\% CI: 1.04-2.90)). Effect modification by sex was identified. The PR in men was 6.00 (95\% CI: 1,98-18.16), while the in women was $\mathrm{PR}=2.11$ (95\%CI: $1.29-3.44)$ in women.

Conclusions Findings reinforce the hypothesis that arboviruses, particularly Chikungunya, are independently associated with depression. Physiological viral action, as well as emotional, behavioural and psychosocial factors may partially justify these findings. Sex differences are notably high and must be further investigated, in order to understand possible mechanisms related to this association.

\section{P-475 SOCIOECONOMIC AND CLINICAL FACTORS ASSOCIATED WITH DEATH AND HOSPITALIZATION OF HEALTH PROFESSIONALS AFFECTED BY COVID-19 IN BRAZIL, 2020}

${ }^{1}$ Rodolfo Corona, Arthur Cunha. ${ }^{1}$ Federal University of Amapá, Brazil

10.1136/OEM-2021-EPI.344

Introduction The new coronavirus pandemic affected the lives of millions of people across the planet in 2020. Brazil, in just a few months, became the epicenter of the pandemic in Latin America. This, due to the absence of vaccines and the ineffective isolation measures adopted. In this context, health professionals stand out as a group with greater exposure and risk of being affected by COVID-19.

Objective To analyze clinical and sociodemographic characteristics associated with death and hospitalization of health professionals due to COVID-19, in addition to calculating the incidence rates per occupation.

Methods We conducted a cross-sectional observational study that used secondary data from the State of Espírito Santo Health Department. COVID-19 cases in healthcare professionals were recorded between February 27 and August 17, 2020 in Espírito Santo, Brazil. Cases with a lack of information were excluded. To compare the sociodemographic and clinical characteristics of health professionals with the clinical outcomes (cure or death) and the need for hospitalization, a bivariate analysis was performed using the $X^{2}$ tests of independence or Fisher's exact test. Data analysis was performed using the SPSS 20.0 software.

Results 75.6\% ( $\mathrm{n}=9,191)$ of the cases were female; the general lethality rate was $0.27 \%$ and the general hospitalization rate was $1.0 \%$. The clinical outcome of death and the occurrence of hospitalization were associated with: male gender, age greater than or equal to 50 years, higher education, fever, difficulty breathing, cough, cardiac comorbidity, diabetes and obesity ( $p<0.05)$. Only the occurrence of hospitalization was associated with: case reported in the metropolitan region of Vitória-ES, runny nose, sore throat, headache and renal comorbidity $(p<0.05)$. The occupation with the highest incidence rate was nurses (16,053 cases/100,000 nurses).

Conclusion The study demonstrated a high frequency of cases in females, low general lethality and high incidence in nurses.

\section{P-476 TRENDS IN TIME SERIES OF COMMUTING ACCIDENTS OF THE MOST AFFECTED ECONOMIC ACTIVITIES IN BRAZIL, 2008 TO 2017}

${ }^{1}$ Rodolfo Corona, Arthur Cunha, Giovana Carvalho, Emerson Castilho-Martins. ${ }^{1}$ Federal University of Amapá, Brazil

\subsection{6/OEM-2021-EPI.345}

Introduction Accidents at work are an important public health problem in Brazil. Among them, commuting accidents stand out, which can be defined as a type of work accident suffered on the way from home/place from meal to work or vice versa. Its consequences interfere in the national economy and generate suffering for the victim and his family.

Objectives To evaluate the trends of time series (2008-2017) of commuting accidents in the five most affected economic activities and to identify the epidemiological profile of the injured in Brazil.

Method This study is based on official Social Security data. The five most affected economic activities were determined by means of simple sums of absolute numbers. Time series trends were performed using simple time regression models. The epidemiological profile was determined based on the variables of sex and age. The regressions were performed with the SPSS 20.0 software, being considered significant, with reference to the $\mathrm{F}$ test, when $\mathrm{p}$-value $\leq 0.05$.

Results The most affected economic activities were Hospital Care. 